\title{
AN ELEMENTARY PROOF OF BENEDICKS'S AND CARLESON'S ESTIMATES OF HARMONIC MEASURE OF LINEAR SETS
}

\author{
MIKHAIL SODIN
}

(Communicated by Albert Baernstein II)

\begin{abstract}
Benedicks and Carleson proved sophisticated estimates of harmonic measure of linear sets which have found applications in approximation theory and harmonic analysis. We give an elementary proof of these estimates. This proof allows us to relax the assumptions and strengthen estimates.
\end{abstract}

Let $Q_{w}(h)$ be the square in the complex plane with center $w$ and sidelength $h$, and let $Q(h)=Q_{0}(h)$. We will denote by $C$ absolute positive constants, the values of which may vary, and by $|E|$ the linear measure of a set $E$.

In the paper [Bel] Benedicks proved the estimate of harmonic measures

Lemma 1 (Benedicks). Let $E \subset \mathbb{R} \cap Q(r)$ be a closed set such that

$$
\left|E \cap Q_{x}(h)\right| \geq \delta h
$$

for some $\delta>0, h<r$, and all $x,|x| \leq r-h$. Then

$$
\omega(0) \leq \frac{C h}{\delta^{3} r}
$$

where $\omega(z)=\omega(z, \partial Q(r), Q(r) \backslash E)$ is the harmonic measure of $\partial Q(r)$ with respect to $Q(r) \backslash E$ at the point $z$.

Benedicks's proof of this estimate is based on a sophisticated iterative scheme (see [Be1, Lemma 8]). Carleson pointed out another estimate of this sort that may be proved by Benedicks's method (see [C, §6] or [K, §VIIIA1]).

Lemma 2 (Carleson). Let $E \subset \mathbb{R}$ be such a closed set that (1) is fulfilled with some $h<\infty, \delta>0$. Then

$$
\omega(x, E \cap[-h, h], \mathbb{C} \backslash E) \leq \frac{K(\delta, h)}{1+|x|^{2}} .
$$

These estimates have found important applications in harmonic analysis [K, $\S$ VIII].

Received by the editors April 20, 1992 and, in revised form, November 2, 1992; presented to the AMS regional meeting at Springfield, Missouri, March 20, 1992.

1991 Mathematics Subject Classification. Primary 30C85, 31A15.

Key words and phrases. Harmonic measure, positive harmonic function in half-plane, capacity. 
Our purpose is to give an elementary proof of these estimates that allows us to relax their assumptions and slightly strengthen their assertions. This proof is based on a lemma that was proved recently in [LLS] though in essence it goes back to Schaeffer [S] and Akhiezer and Levin [AL].

Before stating this lemma we introduce some notation. Let $E$ be a closed set contained in the strip $\{|\Im z|<1 / 4\}$, and let $\mathfrak{K}(E)$ be the class of functions which are subharmonic in $\mathbb{C}$ with order at most one and type not exceeding one:

$$
\limsup _{|z| \rightarrow \infty} \frac{u(z)}{|z|} \leq 1,
$$

and are nonpositive on $E$. By $\operatorname{cap}(\cdot)$ we denote logarithmic capacity.

Lemma 3 [LLS]. Suppose that for every $x \in \mathbf{R}$ and for some $\delta>0$

$$
\operatorname{cap}\left(E \cap Q_{x}(1 / 2)\right) \geq \delta \text {. }
$$

Then

$$
u(x) \leq C \log \left(\frac{1}{\delta}\right)
$$

for every function $u \in \mathfrak{K}(E)$ and every real $x$.

For the convenience of the reader we recall the proof from [LLS], especially since the result of [LLS] is more general and contains technical details which are unnecessary here.

\section{Proof of Lemma 3}

Let $E_{N}=E \cup\{|x| \geq N\}$. We will prove that (4) holds for every function $u \in \mathfrak{K}\left(E_{N}\right)$ and every real $x$ with an absolute constant $C$ (that does not depend on $N)$. Then, by the usual Phragmén-Lindelöf Principle, functions from $\mathfrak{K}\left(E_{N}\right)$ must satisfy the estimate

$$
u(z) \leq|\Im z|+C \log (1 / \delta)
$$

in the whole complex plane, and by a normal family argument we may conclude that functions from $\mathfrak{K}(E)$ also satisfy estimate (5) and, therefore, estimate (4).

Thus fix a function $u \in \mathfrak{K}\left(E_{N}\right)$ and set

$$
M_{u}=\max _{x \in \mathbb{R}} u(x)<\infty .
$$

Now we choose a point $x_{0} \in \mathbb{R}$ such that $u\left(x_{0}\right)=M_{u}$ and consider the square $Q_{0}=Q_{x_{0}}(1 / 2)$. Again by the Phragmén-Lindelöf Principle

$$
u(z) \leq M_{u}+|\Im z| \leq M_{u}+1 / 4, \quad z \in \partial Q_{0},
$$

and by the Maximum Principle

$$
M_{u}=u\left(x_{0}\right) \leq \omega\left(x_{0}, \partial Q_{0}, Q_{0} \backslash E\right)\left(M_{u}+1 / 4\right) ;
$$

thus,

$$
M_{u} \omega\left(x_{0}, Q_{0} \cap E, Q_{0} \backslash E\right) \leq 1 / 4 .
$$

So all that remains is to use the standard estimate

$$
\omega\left(0, Q_{0} \cap E, Q_{0} \backslash E\right) \geq c \log ^{-1}\left(\frac{1}{\operatorname{cap}\left(Q_{0} \cap E\right)}\right)
$$

(see, for example, [R, Lemma 1.4.1] or [LLS, Lemma 3]). Lemma 3 is proved. 
Corollary 1 [LLS]. Suppose that for every real $x$ and for some $h<\infty$ and $\delta>0$

$$
\operatorname{cap}\left(\frac{1}{2 h}\left(E \cap Q_{x}(h)\right)\right) \geq \delta
$$

Then

$$
u(x) \leq C h \log (1 / \delta)
$$

for every function $u \in \mathfrak{K}(E)$ and every $x \in \mathbb{R}$. Here $(1 / 2 h)\left(E \cap Q_{x}(h)\right)$ is the image of $E \cap Q_{x}(h)$ under the linear map $x^{\prime} \rightarrow x+\left(x^{\prime}-x\right) / 2 h$.

For the proof consider the function $u(2 h z) / 2 h$ and apply Lemma 3 .

Remark 1. It was proved in [LLS] that condition (6) in Corollary 1 cannot be weakened. Namely, for every subset $E$ of the real axis that is not uniformly dense by capacity, there exists a subharmonic function $u \in \mathfrak{K}(E)$ unbounded on the real axis.

Remark 2. Baernstein [Ba] and Fryntov [F] proved independently that if in the assumption of Lemma 3 inequality (3) is replaced by

$$
\left|E \cap Q_{x}(1 / 2)\right| \geq \delta
$$

then the best possible value of the right-hand side of (4) is

$$
\frac{1}{2 \pi} \log \left(\cot \frac{\pi \delta}{4}\right) \text {. }
$$

Their proof is based on symmetrization techniques. It seems interesting to find whether this constant is the best possible in the conditions of Lemma 3.

Our proof of Benedicks's and Carleson's estimates is based on a comparison of corresponding harmonic measures with subharmonic majorant

$$
v_{E}(z)=\sup _{u \in \mathfrak{K}(E)} u(z)
$$

which is a positive harmonic function in $\mathbb{C} \backslash E$ with zero values on $E$.

\section{BENEDICKS-TYPE ESTIMATE}

Lemma 4. Let $r>0$ and $E \subset Q(r) \cap \mathbb{R}$ be a closed set such that

$$
\operatorname{cap}\left(\frac{1}{2 h}\left(E \cap Q_{x}(h)\right)\right) \geq \delta
$$

for some $\delta>0, h<r$, and for all $x,|x| \leq r-h$. Then

$$
\omega(0) \leq \frac{C h}{r} \log \left(\frac{1}{\delta}\right)
$$

where $\omega(z)=\omega(z, \partial Q(r), Q(r) \backslash E)$.

Proof. We will assume without loss of generality that each point of $E$ is regular for Dirichlet Problem in $\mathbb{C} \backslash E$. 
By an elegant and elementary lemma of Benedicks ([Be1, Lemma 7] or [K, p. 436]) we have

$$
\omega(0) \leq 2 \omega^{*}(0)
$$

where $\omega^{*}(z)=\omega(z, \partial Q(r) \cap\{|\Im z|=r\}, Q(r) \backslash E)$.

Set $E_{r}=E \cup\{|x| \geq r\}$, and consider the upper envelope of subharmonic functions

$$
v_{E_{r}}(z)=\sup _{u \in \mathfrak{K}\left(E_{r}\right)} u(z) .
$$

This is a positive harmonic function in $\mathbb{C} \backslash E_{r}$ with zero boundary values on $E_{r}$. In particular, this function is harmonic in $Q(r) \backslash E$. The set $E_{r}$ satisfies condition (6) (with $2 h$ instead of $h$ ), so

$$
v_{E_{r}}(x) \leq C h \log (1 / \delta) .
$$

The function $v_{E_{r}}(z)$ has type one and order one, so by the representation of positive harmonic function in upper and lower halfplanes

$$
|\Im z| \leq v_{E_{r}}(z), \quad z \in \mathbb{C} .
$$

Then $r \omega^{*}(z) \leq v_{E_{r}}(z)$ everywhere on $\partial Q(r)$, and both of these functions equal zero on $E \cap Q(r)$. By the Maximum Principle this inequality holds everywhere on $Q(r)$. Thus by (10) and (11)

$$
r \omega(0) \leq 2 r \omega^{*}(0) \leq 2 v_{E_{r}}(0) \leq C h \log (1 / \delta),
$$

which proves the lemma.

\section{Carleson-type estimate}

To prove the Carleson-type estimate of harmonic measure we will need the following elementary

Lemma 5. Let $f_{a}(t)=\min \left(1,|t|^{-a}\right), 0<a<2$, and let

$$
H_{a}(x+i y)=\frac{y}{\pi} \int_{-\infty}^{\infty} \frac{f_{a}(t)}{(x-t)^{2}+y^{2}} d t
$$

be the Poisson integral of $f_{a}$. Then

$$
H_{a}(x+i y) \leq \begin{cases}C|x|^{-a} & \text { for } a<1, \\ C|x|^{-1} \log |x| & \text { for } a=1, \\ C|x|^{-1} & \text { for } a>1,\end{cases}
$$

for $|x| \geq e,|y| \leq|x| / 2$.

Proof. As usual we split the integral in (12) into parts and estimate each term separately. Assume, for example, that $y>0$. Then

$$
\begin{aligned}
H_{a}(x+i y) & =\int_{|t| \leq 1}+\int_{1 \leq|t| \leq|x| / 2}+\int_{|x| / 2 \leq|t|,|x-t| \geq y}+\int_{|x-t| \leq y} \\
& =J_{1}+J_{2}+J_{3}+J_{4} .
\end{aligned}
$$


We have

$$
\begin{gathered}
J_{1} \leq C \frac{y}{|x|^{2}} \leq \frac{C}{|x|} ; \\
J_{2} \leq C y \int_{1 \leq|t| \leq|x| / 2} \frac{d t}{t^{a}|x-t|^{2}} \leq \frac{C y}{|x|^{2}} \int_{1}^{|x| / 2} \frac{d s}{s^{a}} \\
\leq \begin{cases}C|x|^{-a} & \text { for } a<1, \\
C \log |x| & \text { for } a=1, \\
C|x|^{-2} & \text { for } 1<a<2 ;\end{cases}
\end{gathered}
$$

further,

$$
\begin{aligned}
J_{3} & \leq C y \int_{|x| \leq 2 t,|x-t| \geq y} \frac{d t}{|t|^{a}|x-t|^{2}} \leq \frac{C y}{|x|^{a}} \int_{|x-t| \geq y} \frac{d t}{|x-t|^{2}} \\
& =\frac{C y}{|x|^{a}} \int_{y}^{\infty} \frac{d t}{t^{2}} \leq \frac{C}{|x|^{a}}
\end{aligned}
$$

and

$$
J_{4} \leq C y \int_{|x-t| \leq y} \frac{d t}{|x|^{a} y^{2}}=\frac{C}{|x|^{a}} .
$$

Inequality (13) follows immediately from (14)-(17).

Lemma 6 (Carleson-type estimate). Let for every $x \in \mathbb{R}$ and for some $\delta>0$

$$
\operatorname{cap}\left(E \cap Q_{x}(1 / 2)\right) \geq \delta \text {. }
$$

Then for every $x \in \mathbb{R}$

$$
\omega(x, E \cap[-1,1], \mathbb{C} \backslash E) \leq \frac{C \log ^{3}(1 / \delta)}{1+|x|^{2}}
$$

Proof. Let $\omega(z)=\omega(z, E \cap[-1,1], \mathbb{C} \backslash E)$. We will compare $|x| \omega(x)$ with $v_{E}(x)$. Let $|x| \geq e$. Consider the square $Q_{x}=Q_{x}(|x| / 2)$. Again, as in the proof of Lemma 4, we have

$$
\begin{aligned}
|x| \omega(x) & \leq|x| \omega\left(x, \partial Q_{x}, Q_{x} \backslash E\right) \\
& \leq 2|x| \omega\left(x, \partial Q_{x} \cap\{|\Im z|=|x| / 2\}, Q_{x} \backslash E\right) \leq 2 v_{E}(x) \leq \log (1 / \delta)
\end{aligned}
$$

and

$$
\omega(x) \leq \frac{C}{|x|} \log \left(\frac{1}{\delta}\right) .
$$

Now, following Benedicks (see [Be2] or [K, §VIIIA4]) we apply Lemma 5 to improve this estimate. Using Lemma 5 with $a=1$ we obtain

$$
\omega(x+i y) \leq C \log \left(\frac{1}{\delta}\right) \frac{\log |x|}{|x|}, \quad|y| \leq \frac{|x|}{2} .
$$

Thus we may compare

$$
\log ^{-1}\left(\frac{1}{\delta}\right) \frac{|x|^{2}}{\log |x|} \omega(x+i y) \text { with } v_{E}(x+i y)
$$


in $Q_{x}$. We obtain

$$
\omega(x) \leq C \log ^{2}\left(\frac{1}{\delta}\right) \frac{\log |x|}{|x|^{2}} \leq \frac{C \log ^{2}(1 / \delta)}{|x|^{3 / 2}} .
$$

Applying Lemma 5 the second time we get

$$
\omega(x+i y) \leq \frac{C \log ^{2}(1 / \delta)}{|x|}
$$

in $Q_{x}$, and now we may already compare

$$
\log ^{-2}(1 / \delta)|x|^{2} \omega(x+i y) \text { with } v_{E}(x+i y)
$$

in the same square $Q_{x}$. Thus we obtain (18). Lemma 6 is proved.

Remark 3. Estimate (18) may be rewritten in the form

$$
\omega(x) \leq K(E) \frac{v_{E}(x)}{1+|x|^{2}} .
$$

In this form Carleson's estimate holds for more general sets $E$. It seems plausible that estimate (19) is true whenever the subharmonic majorant $v_{E}$ of the class $\mathfrak{K}(E)$ exists. Unfortunately we are able to prove (19) only in the case when $v_{E}(x)$ has "a good majorant" on the real axis. For example, if

$$
v_{E}(x) \leq C\left(1+|x|^{\alpha}\right)
$$

with some positive $\alpha<1$ then the proof given above does not need any changes. (In [LLS] the necessary and sufficient conditions on the set $E$ for (20) were found.)

In connection with (19) we would like to mention the estimate that easily follows from [K, §VIIIC2]

$$
\omega(x, E \cap[-|x| / 2,|x| / 2], \mathbb{C} \backslash E) \leq C v_{E}(x) /(1+|x|)
$$

for all real $x$. This estimate is true whenever the function $v_{E}(x)$ exists.

Remark 4. All arguments may be repeated verbatim for $\mathbb{R}^{n+1}$ with the "real hyperplane" $\mathbb{R}^{n}$ instead of the real axis.

\section{ACKNOWLEDGMENT}

The author thanks Alex Eremenko for the crucial discussion of the link between Lemmas 1 and 3 and David Drasin and Paul Koosis for helpful remarks. This work was done while the author was visiting at Purdue University. He thanks the Mathematics Department of Purdue University for generous hospitality.

\section{REFERENCES}

[AL] N. I. Akhiezer and B. Ya. Levin, Generalizations of Bernstein inequality for derivatives of entire function, Studies in the Modern Problems of Complex Variables, Fizmatgiz, Moscow. (Russian)

[Ba] A. Baernstein, An extremal problem for certain subharmonic functions in the plane, Rev. Mat. Iberoamericana 4 (1988), 199-218.

[Be1] M. Benedicks, Positive harmonic functions vanishing on the boundary of certain domains in $\mathbf{R}^{n}$, Ark. Mat. 18 (1980), 53-72. 
[Be2] _ Weighted polynomial approximation on subsets of the real line, preprint, Uppsala Univ. Math. Dept., 1981.

[C] L. Carleson, Estimates of harmonic measures, Ann. Acad. Sci. Fenn. Ser. A I Math. 7 (1982), 25-32.

[F] A. Fryntov, On extremal problem of the potential theory, Soviet Math. Dokl. 37 (1988), 754-755.

[K] P. Koosis, The logarithmic integral. I, Cambridge Stud. Adv. Math., vol. 12, Cambridge Univ. Press, Cambridge, 1988.

[LLS] B. Ya. Levin, V. N. Logvinenko, and M. L. Sodin, Subharmonic functions of finite degree bounded on subsets of the "real" hyperplane, Adv. Soviet Math. 11 (1992), 181-197.

[R] L. I. Ronkin, Introduction to the theory of entire functions of several variables, Transl. Math. Monographs, vol. 44, Amer. Math. Soc., Providence, RI, 1974.

[S] A. C. Schaeffer, Entire functions and trigonometric polynomials, Duke Math. J. 20 (1953), 77-88.

Department of Mathematics, Purdue University, West Lafayette, Indiana 47907

Current address: Division of Mathematics, Institute for Low Temperature Physics and Engineering, Lenin Pr. 47, Kharkov 310064, Ukraine

E-mail address: sodin@ilt.kharkov. ua 